\title{
The Crocacins: Novel Natural Products as Leads for Agricultural Fungicides
}

Patrick J. Crowley*, lan H. Aspinall, Kevin Gillen, Christopher R.A. Godfrey, Ingrid M. Devillers, Gordon R. Munns, Olivia-Anabelle Sageot, Joe Swanborough, Paul A. Worthington, and John Williams

\begin{abstract}
A short route to the synthesis of analogues of the antifungal but unstable natural product crocacin $D$ was developed. A wide range of compounds were made in which the complex side chain was replaced by simple aromatic units, and many of them showed high activity in a mitochondrial beef heart respiration assay. Some of these compounds were active against plant pathogenic fungi of agricultural importance in glasshouse tests. In order to find compounds with greater stability than the natural crocacins, the photolabile (Z)-enamide was replaced with many different moieties, but none gave rise to useful fungicidal activity.
\end{abstract}

Keywords: Agrochemical $\cdot$ Crocacin $\cdot$ Fungicide $\cdot$ Natural product $\cdot$ Respiration

\section{Introduction}

Natural products have played a significant role in the discovery of new agricultural fungicides because they often have interesting structures, novel biochemical modes of action, and unusual biological activity. However, they also frequently have highly complex structures, are often unstable, and are not always as biologically active as modern commercial pesticides. Nevertheless their novel structures continue to inspire chemists to synthesise simplified analogues with optimised properties such as a broad spectrum of disease control, good persistence of effect and high cost efficacy [1].
In 1994 Profs. Höfle and Reichenbach at GBF in Braunschweig, Germany isolated the new compounds crocacins $\mathrm{A}, \mathrm{B}, \mathrm{C}$, and $\mathrm{D}$ from the myxobacterium Chondromyces crocatus, and identified their structures as the novel ( $Z$ )-enamides $\mathbf{1}, \mathbf{2}, \mathbf{3}$, and 4 [2] (Fig. 1). In further studies crocacins A (1) and D (4)were shown to inhibit the electron transport chain at complex III in a beef heart mitochondrial respiration assay, and to inhibit the growth of several fungi in vitro. Crocacins B (2) and C (3) had no significant biological activity.

The mode of action was of special interest as the important commercial fungicides azoxystrobin (5) and kresoximmethyl (6) [3], analogues of the natural product strobilurin A (7), also inhibit mitochondrial respiration in plant pathogens at complex III [4] (Fig. 2). Modelling studies suggested that the crocacins interacted with the active site of complex III in a different manner to that of the strobilurins.

We obtained samples of crocacins A (1) and D (4) from Profs. Hofle and Reichenbach and confirmed their activity against certain fungi in vitro, and also demonstrated that they were active by foliar spray against some plant pathogenic fungi on small plants. Crocacin D (4) was slightly more active than crocacin A (1). The diseases which were controlled included Plasmopara viticola (vine downy mildew), Phytophthora infestans (potato late blight), Puccinia recondita (wheat brown rust), and
Septoria nodorum (wheat glume blotch). We also confirmed their mode of action as inhibition of mitochondrial respiration at complex III in beef heart and the model fungus Neurospora crassa.

With their novel structures, interesting mode of action and significant fungicidal activity, the crocacins were attractive leads for synthesis, although ambitious targets as agrochemicals due to their relatively complex structures. We therefore decided to embark on a programme of chemistry to see if simpler, more active and more stable compounds could be found.

For practical use on crops in the field, fungicides must have reasonable stability on leaf surfaces so that they can kill incoming fungal spores over a period of 1-2 weeks as well as those that are already present. Both crocacin A (1) and D (4) were shown to have low levels of photostability, with $50 \%$ of the parent compound being lost in 7 and $37 \mathrm{~min}$, respectively, as thin films on glass slides in a simulated sunlight test in the laboratory. This low photostability is not surprising in view of the number of double bonds the crocacins contain, and would undoubtedly lead to very poor persistence of fungicidal activity on plants, severely limiting the activity that these compounds could show in the field.

There was no significant aqueous hydrolysis of the glycine methyl ester group of crocacin $\mathrm{A}(\mathbf{1})$ at $\mathrm{pH} 4$ and 7 at $39^{\circ} \mathrm{C}$, but addition of pig liver esterase under these 
conditions led to rapid generation of the acid, suggesting that the compounds might be easily metabolised in plants or in fungi.

This paper will briefly discuss the range of analogues made to overcome these deficiencies and the chemical approaches to them, and only limited reference will be made to biological results. A more detailed discussion of the structure activity relationships, the extensive use of molecular modelling, and X-ray crystallographic studies will be published elsewhere.

\section{Analogues of Crocacins A (1) and $D(4)$}

\subsection{Design Strategy}

There were three major aims in the design of analogues (Fig. 3). The first was to find compounds with improved fungicidal potency on a wider spectrum of plant pathogens. The second was to increase stability compared to the natural products so that analogues would be able to exhibit fungicidal activity under field conditions. The third was to reduce the complexity of the molecules so that they could be synthesised economically. We chose to concentrate on making analogues of crocacin D (4), which was more active and simpler in structure than crocacin A (1), having only one (Z)double bond in the central section of the molecule, although a small number of crocacin A analogues were made.

An essential element of this strategy was to replace most, if not all, the double bonds by more stable groups, in order to achieve photostability. We hoped that this would not only improve fungicidal activity by increasing persistence, but would also lead to simpler molecules.

We speculated that the $(Z)$-enamide glycine ester (fragments d and e in Fig. 3) might be the key toxophore in crocacins A (1) and D (4), and that the lipophilic side chain with the styryl group (fragment a), the array of four contiguous chiral centres (fragment b) and the diene (fragment c) was essentially 'decoration' which could be replaced by stable aromatic groups. The (Z)enamide was predicted to be quite unstable, and would probably need to be replaced by a more robust group. The glycine ester (fragment e) was also a source of potential degradation through hydrolysis by esterases in plant or fungal cells, and might need to be replaced by groups more resistant to hydrolysis.

Following the logic of these ideas, we derived a set of target structures in which much of the crocacin structure had been changed. The $(Z)$-double bonds were replaced with benzene rings, and the whole

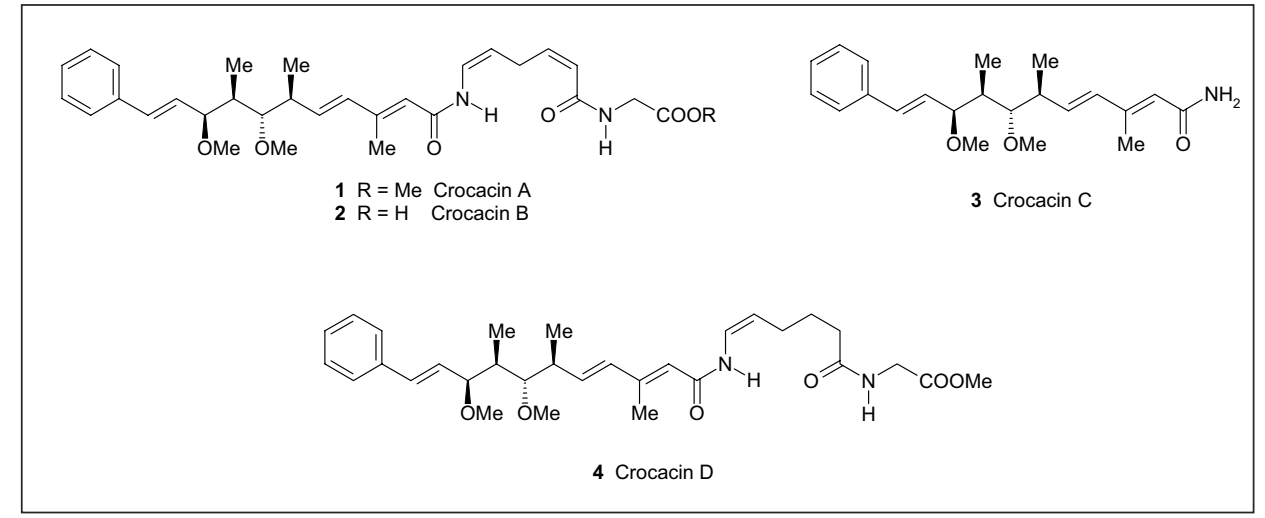

Fig. 1. Structures of crocacins A-D

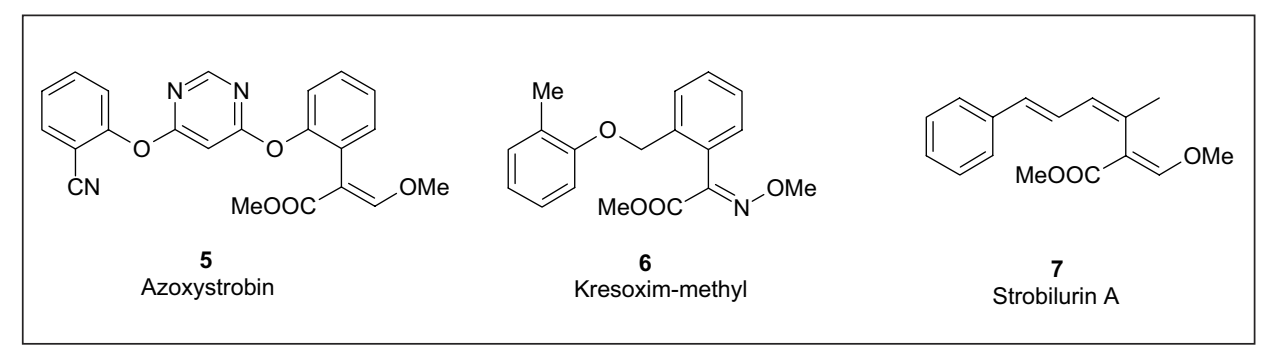

Fig. 2. Structures of strobilurin analogues and strobilurin A (7)

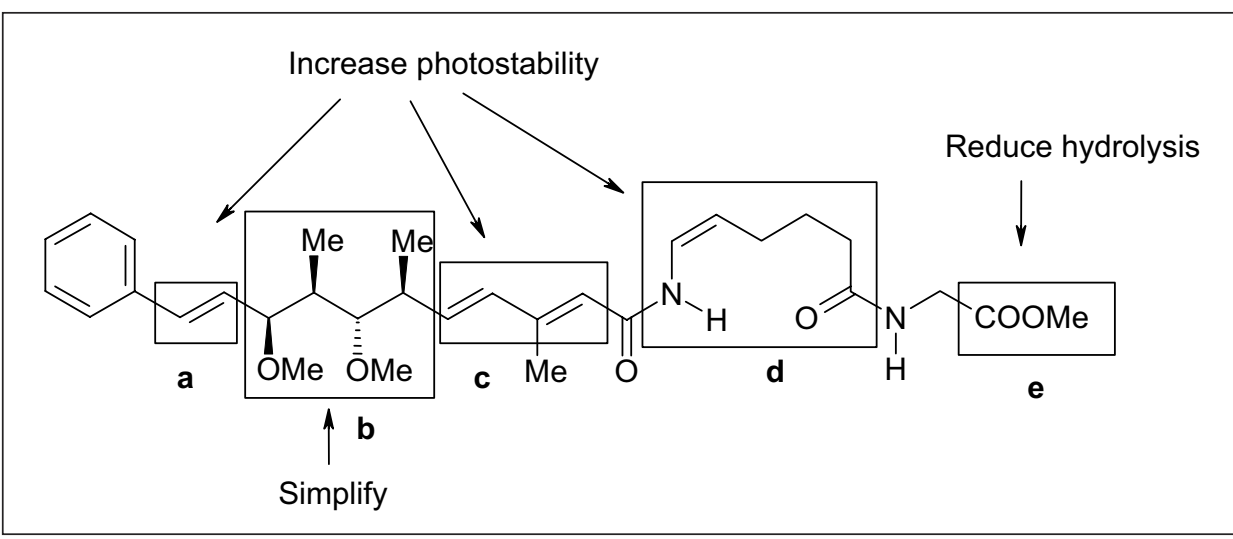

Fig. 3. Plan for design of analogues of crocacin D (4)

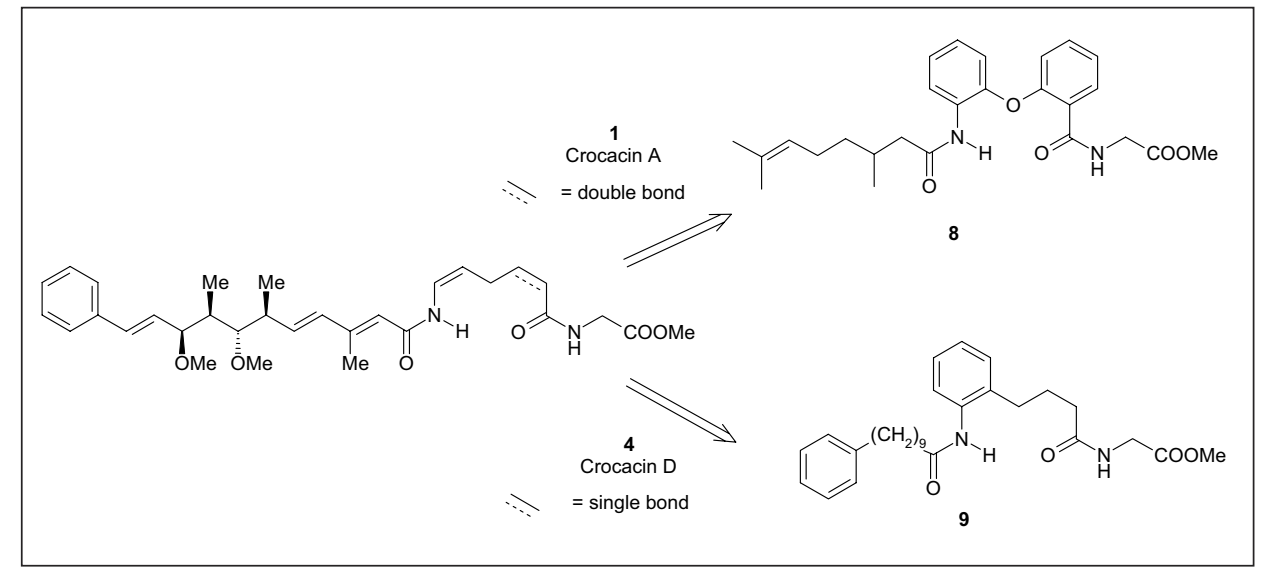

Fig. 4. Analogues of crocacin A and D with double bonds replaced

side chain was replaced by very much simpler groups. Examples of some of the analogues made are compounds 8 and $\mathbf{9}$ (Fig.
4). All compounds were tested on a cascade consisting of a beef heart mitochondrial respiration assay, followed by application 
to fungi on leaf disks, with the most active compounds then being tested on fungi on small plants. Unfortunately neither compounds 8 or 9 or a range of close analogues showed activity in any of these tests.

\subsection{Variation of the Side Chain}

We realised that in pursuing the highly simplified structures of analogues $\mathbf{8}$ and $\mathbf{9}$ we may have made too many changes at once. We therefore decided to adopt a stepwise approach, changing one part of the molecule at a time. We believed that the (Z)-enamide glycine ester was the key toxophore, so we opted to maintain this moiety in our analogues, while replacing the complex side chain with simplified aromatic groups. If this strategy turned out to be successful, we could then seek replacements for the $(Z)$-enamide linker, and mimics for the labile glycine ester.

Indeed, as we had hoped, the complex side chain of crocacins A (1) and D (4) proved not to be necessary for activity, and could be greatly simplified (Table 1). In the first analogues synthesised we replaced the whole side chain with simple n-alkyl chains. An example was compound $\mathbf{1 0}$ which was very active in the beef heart mitochondrial respiration assay, although it was not fungicidal. Benzamides substituted with alkoxy groups such as compound $\mathbf{1 1}$ were similarly good inhibitors of respiration, but were inactive on fungi. However, substituting the benzamide with chains containing aryl groups, such as compound 12, now produced activity on fungi on leaf disks and on plants at a level similar to the crocacins. Benzamides with a 4-substituent were much more active than those substituted in the 3-position, and highest activity was shown by 4 -substituted benzyl analogues such as compound $\mathbf{1 3}$.

\subsection{Replacement of the (Z)-Enamide}

Since these analogues retaining the (Z)enamide group were shown to be quite unstable in laboratory tests, replacing it with alternative but stable linkers was predicted to improve fungicidal activity on plants by increasing the persistence of the compound on leaf surfaces. Many different types of analogue were synthesised (Fig. 5), including some with rings as linking groups such as compounds 14-20, and some with flexible chains such as compounds 21-23. None of these compounds were fungicidal and only the benzoxazolone $\mathbf{2 0}$ had any significant activity on the respiration assay (Table $2)$. The cis-cyclopropane $\mathbf{2 4}$ on the other hand did have weak activity on fungi, but its very poor level of inhibition of respiration casts doubt on this as the mode of action.
Overall we concluded that it is difficult to mimic the rigidity of the $(Z)$-enamide without increasing steric bulk, and that it is likely that space in the active site is very restricted.

\subsection{Mimics of the Glycine Ester}

The glycine methyl ester was found to be important for activity, and simple changes had a marked effect on activity. For example, substitution on the glycine nitrogen or $\alpha$-carbon atoms reduced activity sig- nificantly. Simple esters can be prone to hydrolysis by esterases in plants, and indeed tests using maize cell and liquid fungal cell cultures showed that the methyl ester of several analogues was cleaved very rapidly. Some effort was therefore devoted to looking for groups that might mimic the methyl ester but be more resistant to hydrolysis (Table 3). The $\mathrm{N}$-methylamide $\mathbf{2 5}$ was inactive, but the methyl ketone $\mathbf{2 9}$ showed quite good activity. Certain heterocycles have been shown to be good methyl ester re-

Table 1. Examples of side chain replacements

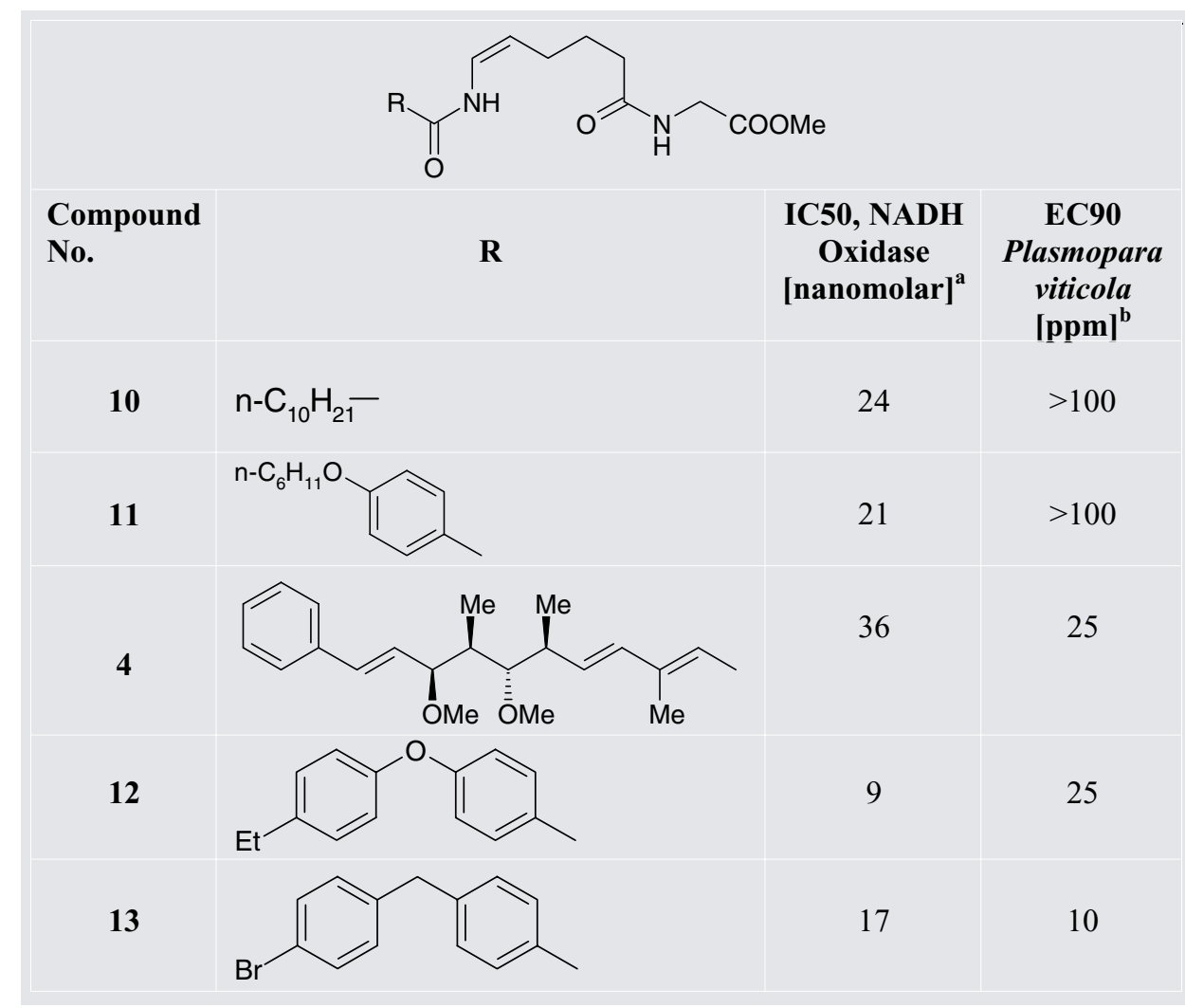

${ }^{a}$ Beef heart mitochondrial preparation. ${ }^{b}$ Foliar spray on small vines.

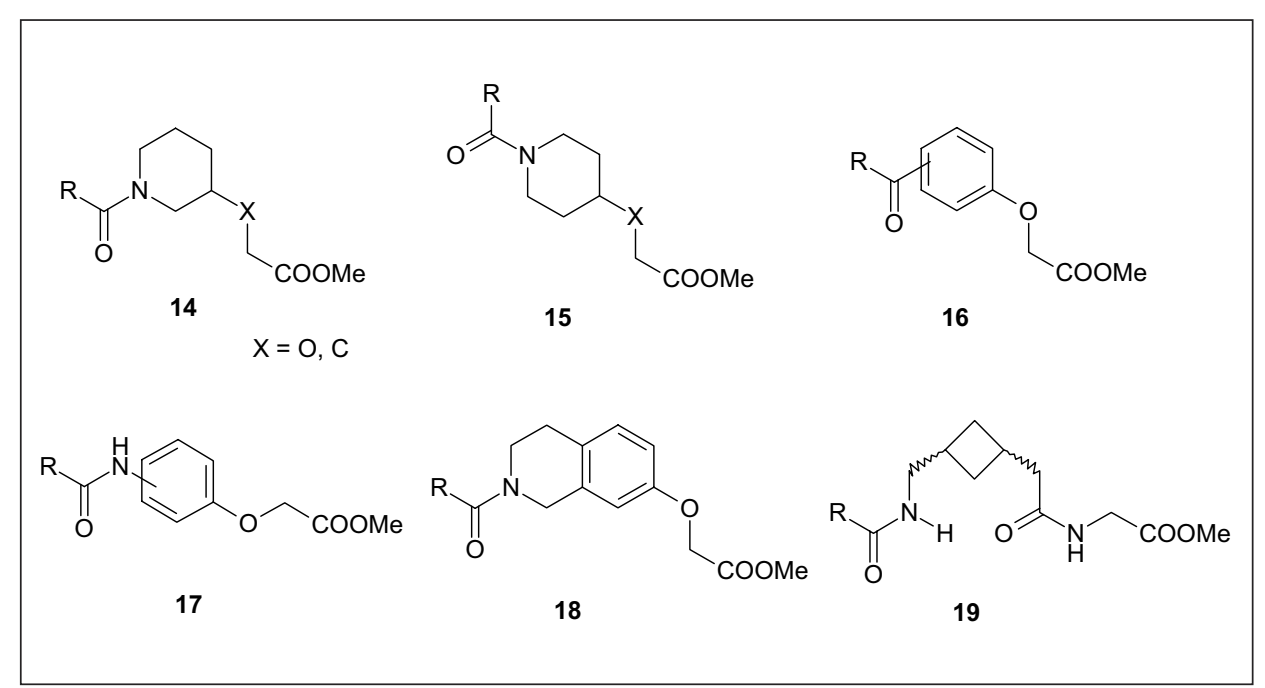

Fig. 5. Examples of (Z)-enamide replacements 
placements [5], and several were tried. The pyrazine $\mathbf{2 8}$ was the best, but although it was resistant to hydrolysis, it was only weakly active in the respiration assay and on fungi.

\section{Biological Activity of Analogues}

The most active analogues controlled a similar spectrum of fungal diseases to the crocacins A (1) and D (4), showing activity in glasshouse tests principally against Plasmopara viticola and Phytophthora infestans on small vine and tomato plants respectively. They also showed high activity on the beef heart respiration assay. However, many compounds that were good inhibitors of respiration were poor or inactive fungicides. The reasons for this are not clear, but may reflect either subtle differences between mammalian and fungal complex III active sites, or differences between compounds in metabolism or stability.

Although the analogues were shown to be more photochemically stable than the natural products, they still had relatively short half-lives on plant surfaces, probably due to the photochemical instability of the $(Z)$-enamide, and none were active enough to progress further.

\section{Synthetic Routes}

In order to make a range of analogues to explore structure activity relationships, we required a synthesis of $(Z)$-enamides. There have been several published syntheses of the complex side chain of the crocacins [6], and recently the first total syntheses of both crocacins A (1) and D (4) have been published [7], using an elegant approach to the (Z)-enamide. However, when our work began in 1995 there was no published chemistry in this area, and there were no good general methods for synthesising ( $Z$ )-enamides. The priority throughout this work was to obtain enough compound to test as rapidly as possible, so most of the chemistry was not optimised, and it is likely that the yields could be improved considerably.

Several attempts were made to synthesise a $(Z$ )-enamide using a lithiation approach. Although alkylation of the model compound $\mathbf{3 0}$ with simple alkyl halides to give 31, following the work of the Tischlers [8] worked well (Scheme 1), no useful products could be obtained from alkylation with groups that could be converted into an ester, for example to give $\mathbf{3 2}$ or $\mathbf{3 3}$.
Table 2. Examples of (Z)-enamide replacements

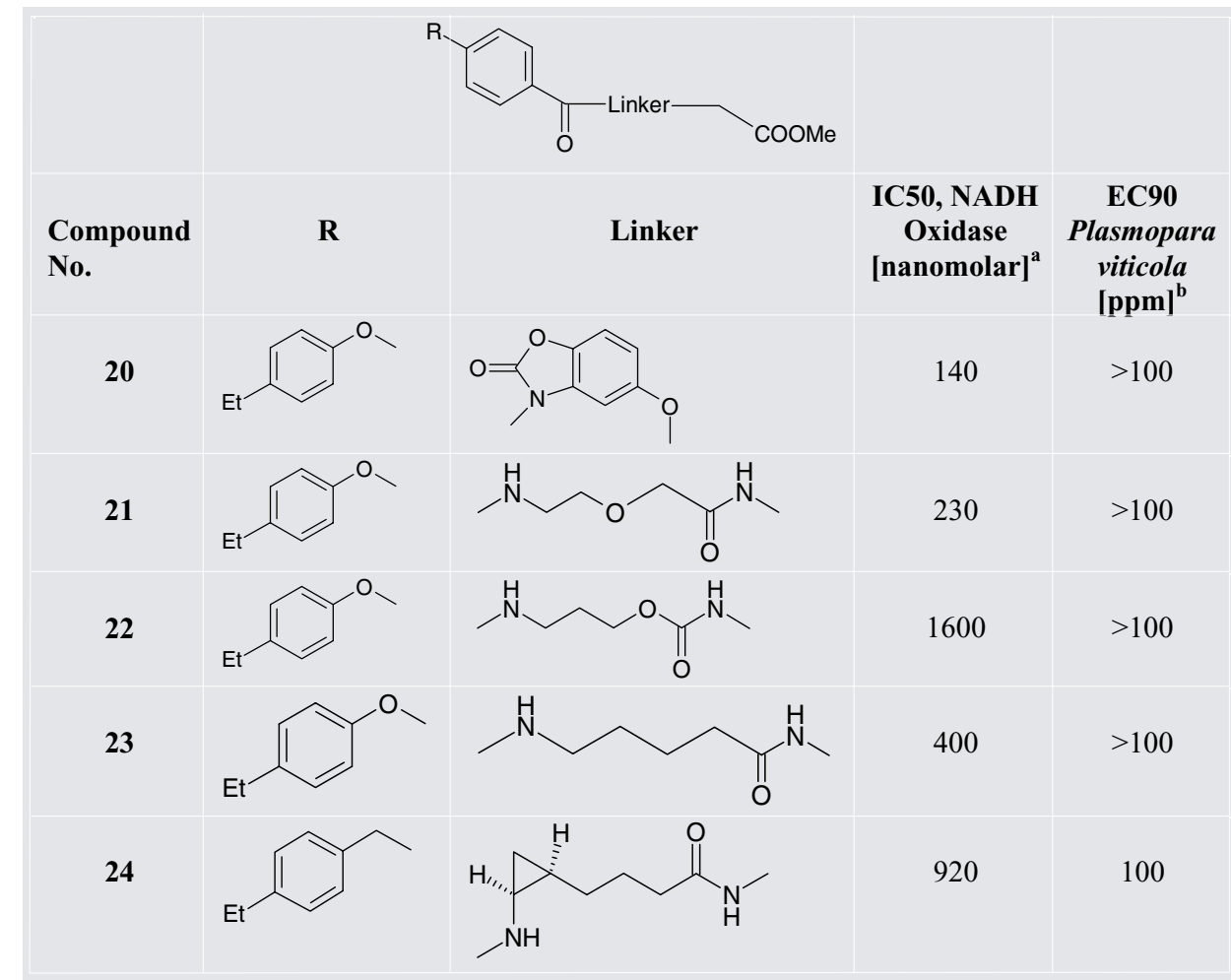

aBeef heart mitochondrial preparation. ${ }^{\mathrm{b}}$ Foliar spray on small vines.

Table 3. Examples of ester mimics<smiles>[2H]Oc1ccc(C(=O)N/C=C\CCCC(=O)O)cc1</smiles>

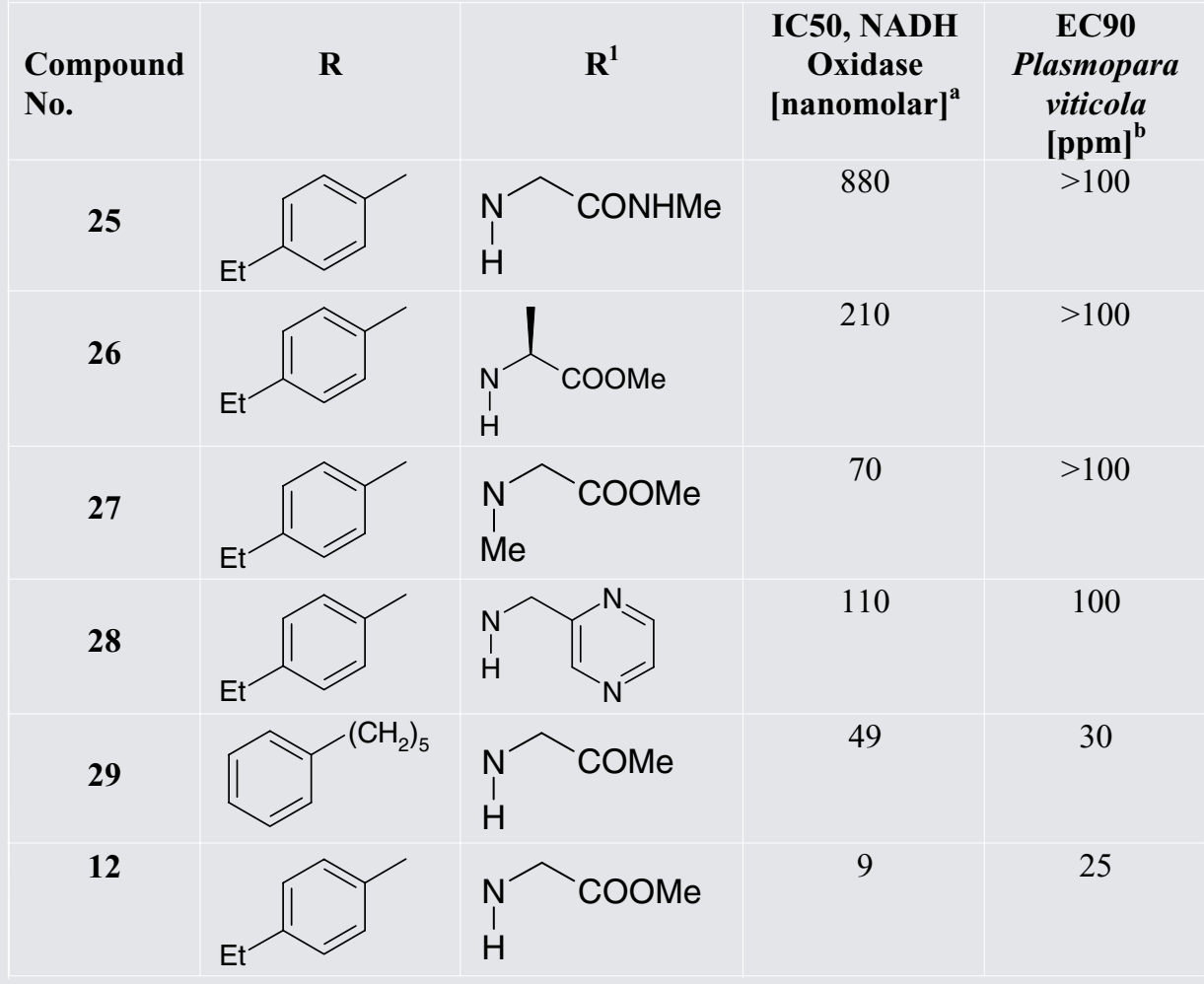

aBeef heart mitochondrial preparation. ${ }^{b}$ Foliar spray on small vines. 
Due to the difficulties of introducing the $(Z)$-enamide we decided to look for starting points where the $(Z)$-double bond was already present, such as the 1,3,4,5-tetrahydro-2H-azepin-2-one (35) (Scheme 2). We envisaged that acylation of compound $\mathbf{3 5}$ to give compound $\mathbf{3 6}$, followed by ring opening with glycine ester, would give the analogues of type $\mathbf{4 1}$ directly. The known 7 ethoxy-1,3,4,5,6,7-hexahydro-2H-azepin2-one (34) was made in good yield by electrolysis of caprolactam in ethanol using a platinum anode. Elimination of ethanol then gave compound 35 [8]. Acylation of the amide nitrogen with acid chlorides was best carried out in the presence of powdered molecular sieves, with no base. However, subsequent ring opening with glycine ester to give compound $\mathbf{4 1}$ or with hydroxide to give compound $\mathbf{3 7}$ proved to be extremely low yielding due to preferential cleavage of the exocyclic acyl group when $\mathrm{R}$ was alkyl.
Protection of the amide nitrogen with Boc to give compound $\mathbf{3 8}$ provided the necessary activation of the ring amide carbonyl group, and reaction with glycine ester now occurred smoothly to give the Boc protected compound 39 in $70 \%$ yield. Unfortunately selective acylation of the Boc amide group to give compound $\mathbf{4 0}$ could not be achieved, and this approach was abandoned.<smiles>C=CCNC(=O)C(C)(C)C</smiles>

30
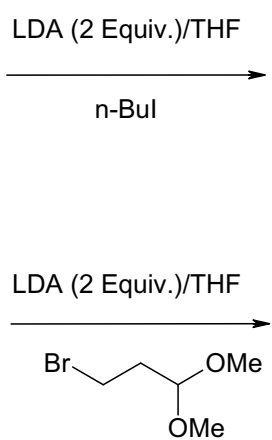

(1) n-BuLi (2 Equiv.)/THF (2) $\mathrm{Cul}$ or $\mathrm{ZnBr}_{2}$<smiles>COC(=O)CCBr</smiles><smiles>CCCCC/C=C\NC(=O)C(C)(C)C</smiles>

31<smiles>COC(CCC/C=C\NC(=O)C(C)(C)C)OC</smiles>

32<smiles>COC(=O)CCC/C=C\NC(=O)C(C)(C)C</smiles>

33 v. low yield

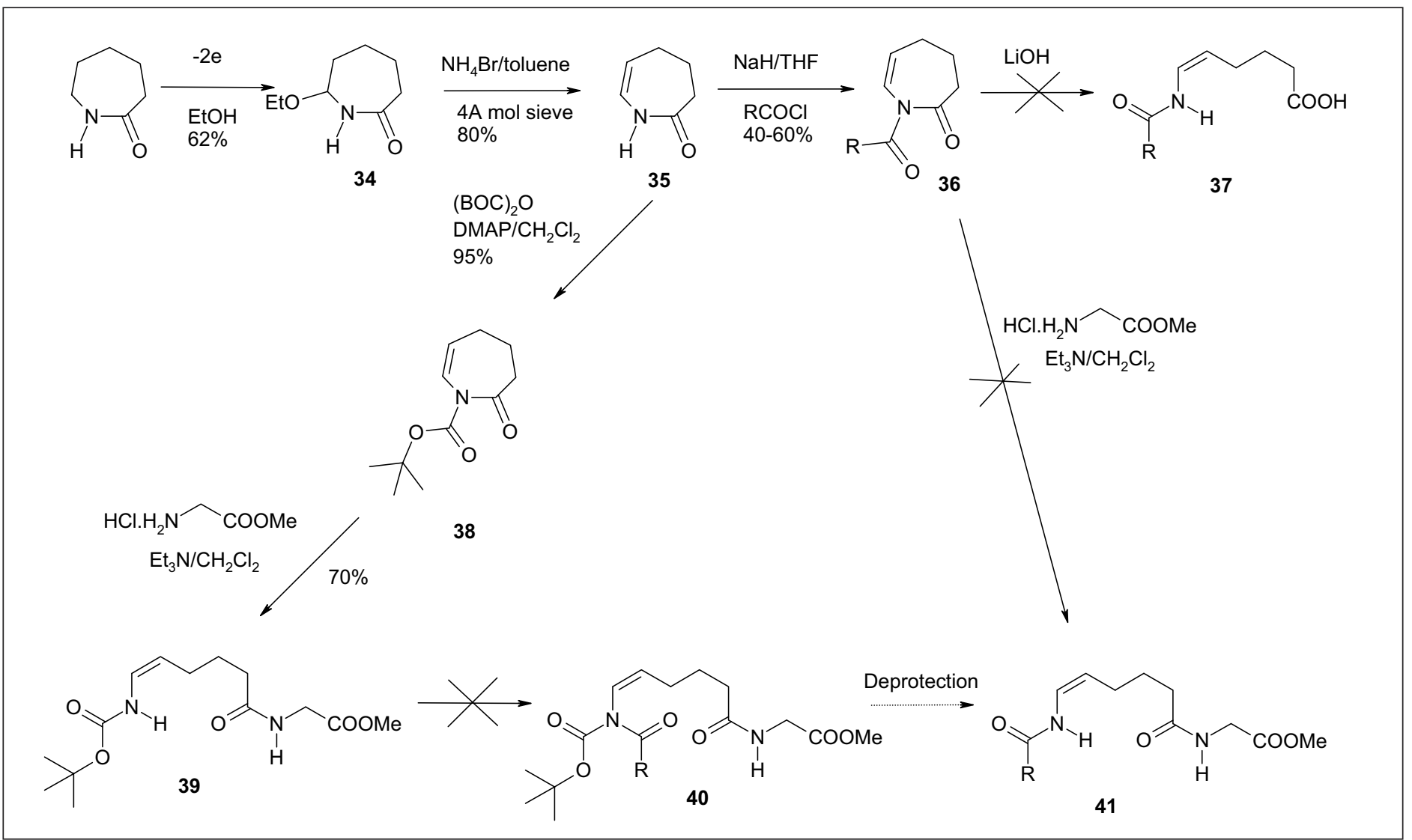

Scheme 2. Synthesis and use of tetrahydroazepinone 35 
An alternative route outlined in Scheme 3 was then tried. The ring in compound $\mathbf{3 8}$ was opened quantitatively with sodium methoxide to give compound $\mathbf{4 2}$, and reaction of the amide anion generated using $\mathrm{LiN}\left(\mathrm{SiMe}_{3}\right)_{2}$ with various acid chlorides gave the imides 43 , although only in 20-30\% yield. Selective deprotection of the Boc group with $\mathrm{Mg}\left(\mathrm{ClO}_{4}\right)_{2}$, again in poor yield, followed by hydrolysis of the ester, gave the key carboxylic acids 37. Simple amide coupling with glycine ester then afforded the desired crocacin analogues 41. Although this sequence gave some valuable analogues where $\mathrm{R}$ was a variety of long chain alkyl groups, the overall yields were very low, and purification of the final products was difficult.

However, for analogues where $\mathrm{R}$ was an aromatic group (Scheme 4), we found a significantly shorter variation of this route, which although giving only moderate yields, was much more convenient and enabled many analogues to be made. The direct acylation of the tetrahydroazepinone 35 with a benzoyl chloride could again be achieved in good yield in the presence of molecular sieves, with no base added. The less reactive benzamide group in $\mathbf{3 6}$ was now less susceptible to hydrolysis, and treatment with hydroxide provided a workable yield of the ring opened product 37 , although variable amounts of acyl cleavage to regenerate the benzoic acid $\mathbf{4 5}$ still occurred. As a matter of practicality, it was convenient to couple the mixture of the carboxylic acids 37 and $\mathbf{4 5}$ with glycine ester, and then separate the desired amide product 41 from the unwanted compound 46. This synthetic route to analogues also worked well when $\mathrm{R}$ was an $\alpha, \beta$-unsaturated group, or a 2- or 3-pyridyl group.

Variations of this route were used to make analogues $\mathbf{4 7}$ where the double bond was replaced by a cyclopropyl group, (Scheme 5), and also where the whole linking group was replaced by a chain containing an oxygen atom as in compound 49. In the latter case an aryl side chain in compound 48 was again required to ensure cleavage at the ring rather than the side chain in the hydrolysis step.

A similar approach was also used to make the benzo-analogues 50 (Scheme 6). A Beckmann rearrangement of tetralone oxime gave the tetrahydrobenzazepinone 50, which as the N-Boc derivative 51, was reacted with glycine ester regioselectively to compound 52. Deprotection then gave the aniline 53, which was acylated with a wide range of carboxylic acids $\mathrm{RCOOH}$, to give analogues 54

The benzylbenzoic ester intermediates required for the most active analogues were

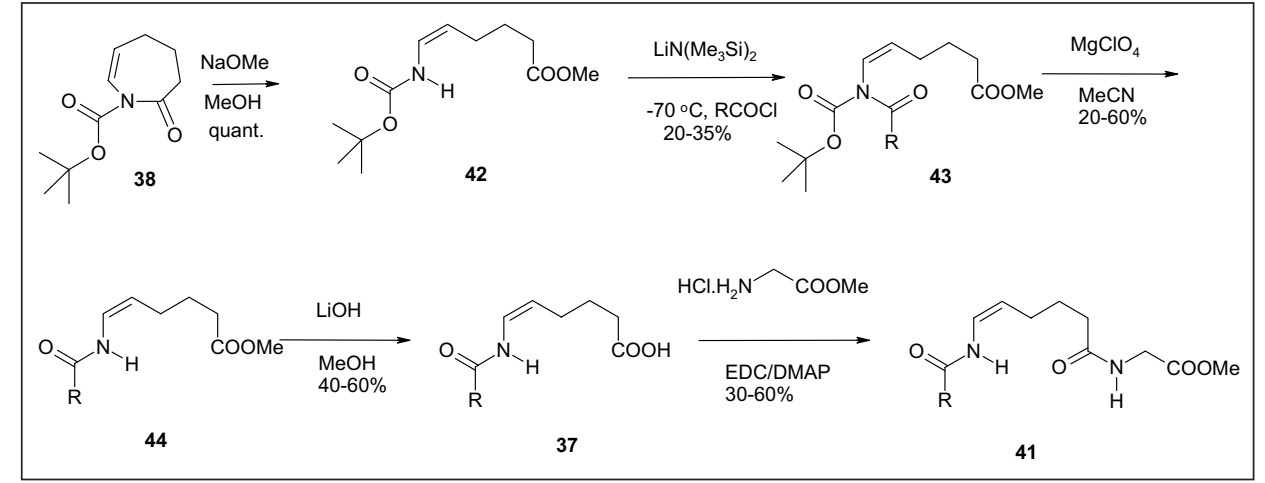

Scheme 3. Synthesis of crocacin analogues with alkyl side chains

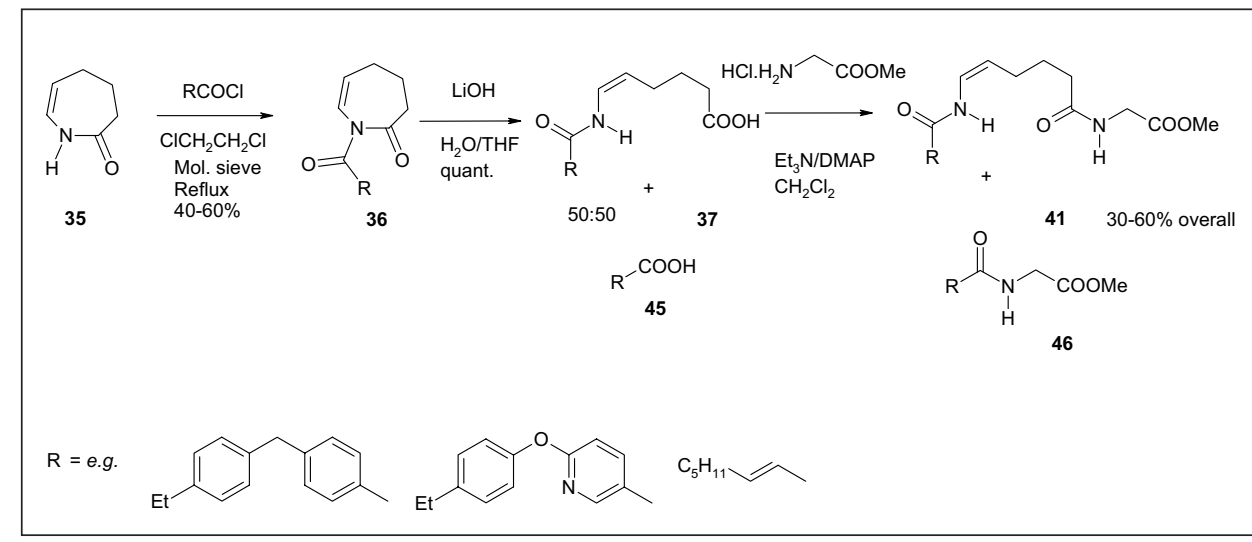

Scheme 4. Synthesis of crocacin analogues with aromatic or unsaturated side chains

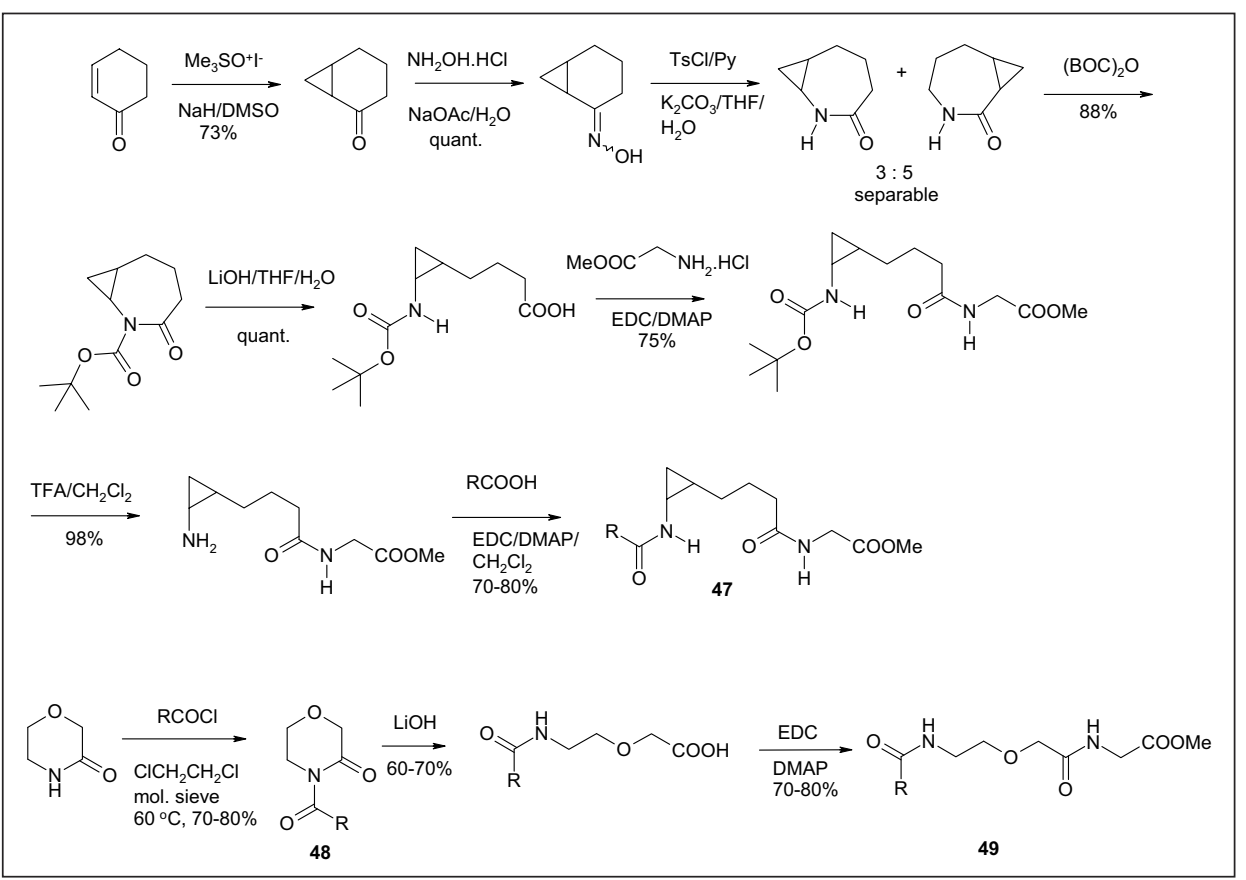

Scheme 5. Synthesis of cyclopropane and flexible chain (Z)-enamide replacements 
made according to Scheme 7. Grignard reaction of ethyl 4-iodobenzoate [10] worked very well to give the key benzhydrol $\mathbf{5 5}$, from which a range of benzylic side chain acids could be made. Reduction to the benzylbenzoic esters 57 was achieved in high yields using an indium trichloride catalysed reaction with dichlorodimethylsilane [11]. Methylation of the benzylic hydroxyl to give $\mathbf{5 8}$ was most efficiently carried out using trimethylsilyldiazomethane in the presence of $\mathrm{HBF}_{4}$ [12]. Oxidation and Wittig reaction to give the methylene compound $\mathbf{5 9}$, followed by triethylsilane reduction gave the methyl derivative $\mathbf{6 0}$.

\section{Conclusions}

We have successfully made a range of analogues of crocacins A (1) and D (4) which were considerably simpler than the natural products. Although a number of these were active against plant pathogenic fungi on plants, none were significantly better, either in activity or stability, than the natural products. Despite considerable effort we were unable to find stable replacements for the central portion of the molecule that gave rise to significant fungicidal activity; the only analogues that were active retained the photochemically vulnerable (Z)-enamide group. We believe that space in the active site where the $(Z)$-enamide fits is probably restricted, so that any change to this part of the structure reduces binding of the whole molecule. Analogues of the crocacins are therefore unlikely to be sufficiently active or stable to make useful agricultural fungicides.

\section{Acknowledgments}

Prof. G Höfle and Prof. H. Reichenbach (both of GBF, Braunschweig, Germany) for providing samples of crocacin $A(\mathbf{1})$ and crocacin $D$ (4), Dr L. May and Mrs F. Dupen (both Syngenta) for glasshouse fungicidal activity tests, T.E.M. Fraser (Syngenta) for measurements of physical properties and stabilities, and Miss J. Phillips (Syngenta) for measurements of inhibition of mitochondrial beef heart respiration.

Received: September 15, 2003

[1] C.R.A. Godfrey, in 'Agrochemicals from Natural Products', Ed. C.R.A. Godfrey, Marcel Dekker, New York, 1994, p. 312.

[2] B. Kunze, R. Jansen, G. Höfle, H. Reichenbach, J. Antibiot. 1994, 47, 881.

[3] J.M. Clough, C.R.A. Godfrey, J.R. Godwin, R.S.I. Joseph, C. Spinks, Pesticide Outlook 1996, 4, 16.

[4] P.M. Wood, D.W. Hollomon, Pest Management Science 2003, 59, 499 .

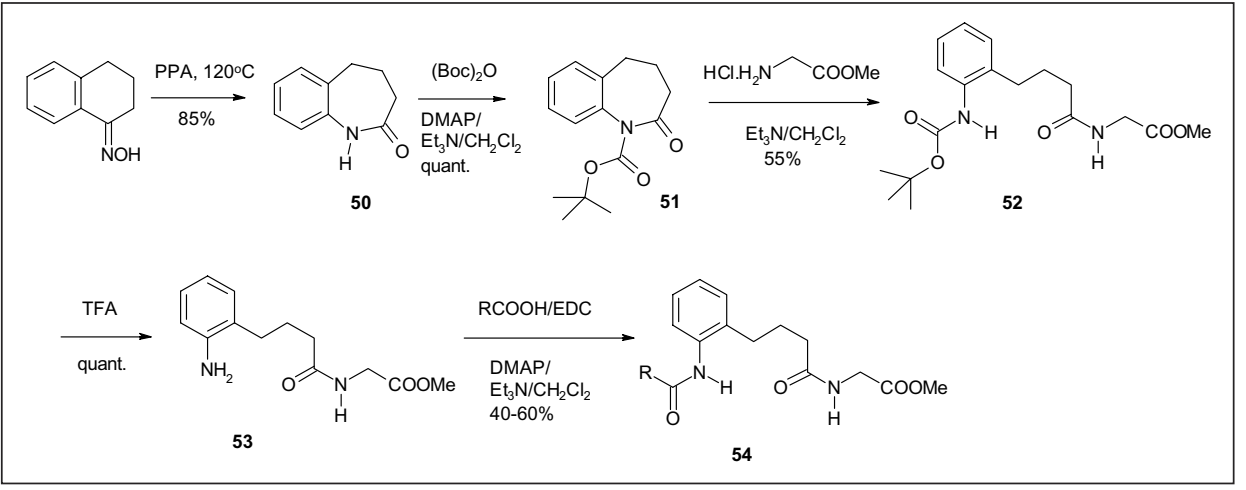

Scheme 6. Synthesis of crocacin analogues with the (Z)-enamide replaced by a benzene ring

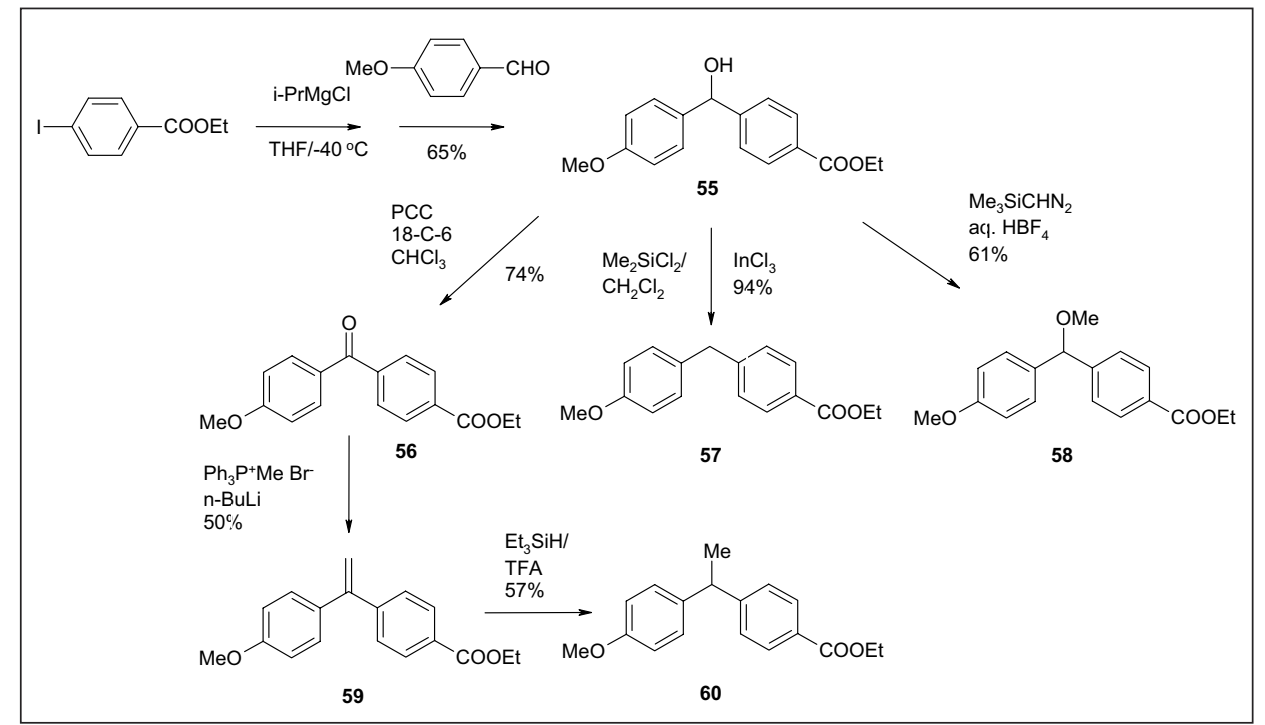

Scheme 7. Synthesis of benzylbenzoic acid side chains

[5] R.T. Lewis, T. Richard, A.M. Macleod, K.J. Merchant, F. Kelleher, I. Sanderson, R.H. Herbert, A. Cascieri, S. Sadowski, R.G. Ball, K. Hoogsteen, J. Med. Chem. 1995, 38, 923; R. Jansen, P. Washausen, B. Kunze, H. Reichenbach, G. Höfle, Eur. J. Org. Chem. 1999, 1085; P. KrogsgaardLarsen, E. Falch, P. Sauerberg, S.B. Freedman, H.L. Lemboel, E. Meier, Trends in Pharmacological Sciences, 1988, (Suppl.), 69.

[6] a) J.T. Feutrill, M.J. Lilly, M.A. Rizzacasa, Org. Lett. 2000, 2, 3365; b) T.K. Chakraborty, S. Jayaprakash, Tet. Lett. 2000, 42, 497; c) L. Dias, L.G. de Oliveira, Org. Lett. 2001, 3, 3951.

[7] a) T.K. Chakraborty, P. Laxman, Tet. Lett. 2002, 43, 2645; b) T.K. Chakraborty, P. Laxman, Tet. Lett. 2003, 44, 4989.

[8] N. Tischler, M.H. Tischler, Tet. Lett. 1978 , 3407.

[9] M. Mitzlaff, K. Warning (Hoechst AG) DE $2718666,1978$.

[10] P. Knochel, L. Boymond, M. Rottlander, G. Cahiez, Angew. Chem., Int. Ed. 1998, 37, 1701

[11] A. Baba, T. Miyai, M. Ueba, Synlett 1999, 182.

[12] T. Aoyama, T. Shioiri, Tet. Lett. 1990, 31, 5507. 